\title{
Optical conductivity of strongly correlated electron systems
}

\author{
R. Eder ${ }^{1}$, P. Wrobel ${ }^{2}$, and Y. Ohta ${ }^{3}$ \\ ${ }^{1}$ Department of Applied and Solid State Physics, University of Groningen, \\ 9747 AG Groningen, The Netherlands \\ 2 Institute for Low Temperature and Structure Research, \\ 50-950 Wroclaw, Poland \\ ${ }^{3}$ Department of Physics, Chiba University, Chiba 263, Japan
}

(August 9, 2018)

\begin{abstract}
We present an exact diagonalization study of the frequency and wave vector dependent conductivity $\sigma(\boldsymbol{q}, \omega)$ in small clusters of $2 D t-J$ model. Unlike the related dynamical density correlation function, $\sigma(\boldsymbol{q}=0, \omega)$ in the underdoped regime has the exchange constant $J$ as its characteristic energy scale and is dominated by a resonance-like excitation with frequency $\sim 1.7 \mathrm{~J}$. We interpret this as transition to a $p$-like excited state of a spin-bag type quasiparticle (or, alternatively, a tightly bound spinon-holon pair) and show that a simple calculation based on the string picture explains the numerical results semiquantitatively. For doping levels $\geq 25 \% t$ remains the only energy scale of $\sigma(\boldsymbol{q}=0, \omega)$.

74.20.-Z, 75.10.Jm, 75.50.Ee
\end{abstract}

Electronic excitations at finite frequency seem to be a common feature of doped Mott-Hubbard insulators, and are observed experimentally in the optical conductivity of cuprate superconductors [1], as well as in numerical studies of $2 \mathrm{D}$ strong-correlation models 2, 3 . 3 . It is the purpose of the present paper to present a systematic study of the optical response function and its dependence on both, parameter values and hole concentration, for the standard $t-J$ model. This model is supposed to describe the low energy physics of the $\mathrm{CuO}_{2}$ planes, at least for energies which are below the 'binding energy' of a Zhang-Rice singlet; the latter may be estimated to be $\approx 1 \mathrm{eV}$. As a key result, the optical conductivity $\sigma(\boldsymbol{q}=0, \omega)$ in the underdoped regime is shown to be dominated by a single excitation which has the exchange constant $J$ as its relevant energy scale, in contrast to e.g. the behaviour seen for the closely related (see below) dynamical charge correlation function 田. We show that a simple calculation based on the string picture can explain this dominant excitation as a transition to a $p$-like excited state of a spin bag-like quasiparticle or, alternatively, a tightly bound spinon-holon pair. We study the finite frequency optical response, defined as

$$
\sigma_{\alpha}(\mathbf{q}, \omega)=\sum_{\nu \neq 0} \frac{1}{\omega}\left|\left\langle\psi_{\nu}\left|j_{\alpha}(\mathbf{q})\right| \psi_{0}\right\rangle\right|^{2} \delta\left(\omega-\left(E_{\nu}-E_{0}\right)\right),
$$

where $\left|\psi_{\nu}\right\rangle\left(E_{\nu}\right)$ denotes the $\nu^{t h}$ eigenstate (eigenenergy) of the system (in particluar $\nu=0$ denotes the ground state). Also, $j_{\alpha}$ with $\alpha=x, y$ denotes a component of the current operator

$$
\boldsymbol{j}(\boldsymbol{q})=i \sum_{m, n} t_{m n} e^{i \boldsymbol{q} \cdot\left(\boldsymbol{R}_{m}+\boldsymbol{R}_{n}\right) / 2}\left[\boldsymbol{R}_{m}-\boldsymbol{R}_{n}\right] \hat{c}_{m, \sigma}^{\dagger} \hat{c}_{n, \sigma} .
$$

In the present study we restrict ourselves to the finitefrequency response and disregard the 'Drude peak' at $\boldsymbol{q}=0$ and $\omega=0$. The latter is absent in finite clusters with periodic boundary conditions (as used in the present study), so that its weight $D$ can only be inferred indirectly via a sum rule:

$$
\frac{-E_{k i n}}{4 N}=\frac{D}{2 \pi e^{2}}+\frac{1}{N} \int_{0^{+}}^{\infty} d \omega \sigma_{x}(\boldsymbol{q}=0, \omega) .
$$

The Drude weight $D$ and its dependence on hole doping and parameter values have been studied previously by various authors. In $2 \mathrm{D}$ systems and hole concentrations $\delta \leq 0.25$ to good approximation $D \sim \delta$, with the constant of proportionality being nearly independent of $J / t$ [5]. Since $D$ originates from the free acceleration of charge carriers in an applied electric field, this result suggests a carrier number given by the number of doped holes, which is consistent with a variety of experiments [6]. In a noninteracting single band model the Drude part is the only contribution to $\sigma(\boldsymbol{q}=0, \omega)$; by contrast, the finite frequency part of $\sigma(\boldsymbol{q}=0, \omega)$ is a special feature of correlated systems, and the excitations which are probed by this part of the correlation function have not yet been identified. In the present study we focus exclusively on this feature of the frequency dependent conductivity.

To begin with, Figure 1 shows the 'dispersion' of the current correlation function $\omega \cdot \sigma(\boldsymbol{q}, \omega)$ for different $J / t$. The 'dominant features' do not change appreciably with $J$, i.e. the energy scale of the correlation function is $t$ (an exception is the largest peak in the $(\pi / 3, \pi / 3)$ spectrum, which seems to scale with $J$ for $J \geq 1$ ). This fact as well as the dispersion of the spectral weight, which resembles a 'smeared out' free electron band of width $\sim 8 t$, is to be expected on the grounds of analogous results for the dynamical charge correlation function (DCF) 传. For e.g. $\boldsymbol{q}$ parallel to $(1,0)$ one has $\left[H, n_{\boldsymbol{q}}\right]=2 \sin \left(q_{x} / 2\right) j_{x}(\boldsymbol{q})$, so that the current correlation for finite momentum transfer 
$\boldsymbol{q}$ is closely related to the DCF, and in principle provides no new information. This is not the case, however, for the optical conductivity, $\sigma(\boldsymbol{q}=0, \omega)$, so that we henceforth consider only case $\boldsymbol{q}=0$ and drop the wave vector $\boldsymbol{q}$ for simplicity.

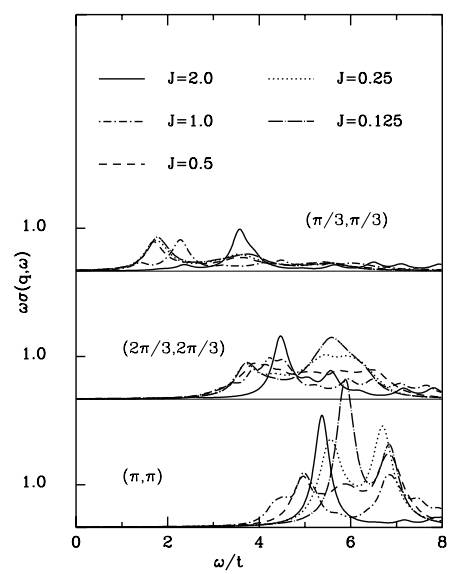

FIG. 1. Current correlation function $\omega \cdot \sigma(\boldsymbol{q}, \omega)$ for different momenta along the $(1,1)$ direction in the 18 -site cluster, with two holes. $\delta$-functions are replaced by Lorentzians of width $0.5 t$

Then, $\sigma(\omega)$ is shown in Figure 2 for 1 and 2 holes and different $J$, for all available cluster sizes $N$ (except for 1 hole in 10 sites, $J=0.125$, which already is in the fully polarized Nagaoka state). Comparing with the spectra in Figure 11 which correspond to large momentum transfer, such as $(\pi, \pi)$, some substantial differences are obvious: for a single hole as well as for two holes and larger values of $J$ the most prominent feature is a pronounced maximum of intensity at the lower edge of the spectra, whose energy scales with $J$. This maximum seems to have a more resonance-like character (i.e. a substantial 'broadening') for two holes and smaller $J$; even in this case, however, the spectra do have a $J$-dependent offset which is nearly the same as for the single hole. The 16-site cluster is exceptional, in that the peak still is very pronounced even for two holes, which may be an effect of its special geometry. As mentioned above, for the smallest momentum transfer, $(\pi / 3, \pi / 3)$, and large $J$ a similar scaling with $J$ can be seen also in Figure 1. Since $\sigma(\boldsymbol{q}, \omega)$ ultimately must be a continuous function of $\boldsymbol{q}$, there has to be a crossover of energy scales from small to large $\boldsymbol{q}$. The wave vector where this crossover occurs apparently depends on $J$ and probably is smaller than $(\pi / 3, \pi / 3)$ for $J \leq 0.5$.

Next, the evolution of the optical conductivity with doping is shown in Figure 3 . There is a rather obvious crossover between the low doping region with a $J$ dependent offset, and the higher doping region where $t$ is the only remaining energy scale. For 'physical' values of $J$, i.e. $J \approx 0.4$, the two-hole spectra in 16,18 and 20 sites(corresponding to $\delta=0.1-0.125)$ still show the
$J$-dependent offset, wheras already at $\delta=0.2(2$ holes in 10 sites) no more more scaling with $J$ can be seen, so that the critical concentration $\delta_{c}$ falls between these two values: $0.125<\delta_{c}<0.2$. Similarly one may estimate that $0.2<\delta_{c}<0.25$ for $J=1$, and $\delta_{c} \approx 0.25$ for $J=2$. It is tempting to speculate that the crossover for physical values is close to the 'optimal doping', in the the cuprates, $\delta=0.15$ but it should be noted that exact diagonalization allows to vary the hole concentration only in rather coarse steps, so that it is difficult to give a really accurate estimate for $\delta_{c}$. We note that such a crossover is also quite consistent with analogous results for spin and charge correlation functions [4]. Moreover the doping dependence of the

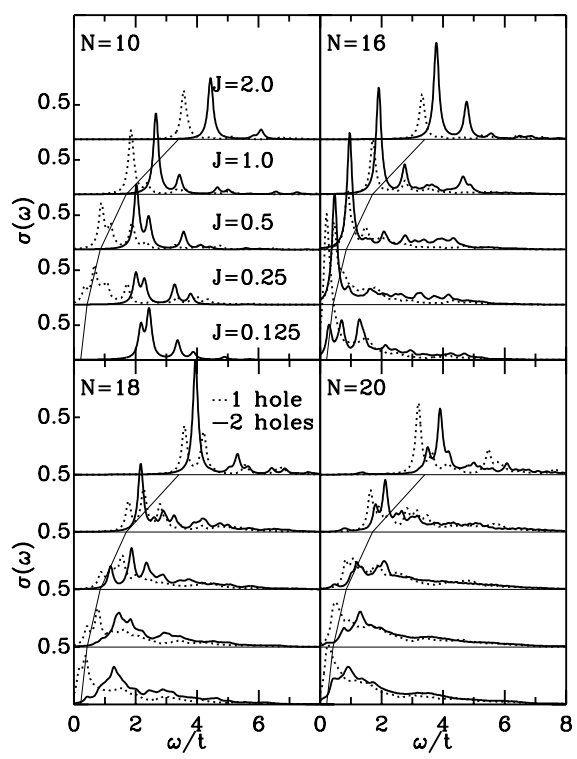

FIG. 2. Optical conductivity $\sigma_{x}(\omega)$ for low hole concentrations in different cluster size $N$. The thin lines indicate the points $\omega=1.7 \mathrm{~J}$, Lorentzian broadening $0.1 t$.

Drude weight changes from the strong-correlation form $D \propto \delta$ to essentially free electron-like behaviour at approximately the same hole concentration [5]. All of this indicates that a profound reconstruction of the electronic structure takes place at this concentration.

We focus on the physically more interesting low doping region and proceed to an interpretation of the dominant ' $J$-peak' in $\sigma(\omega)$. The similarity between the single and two hole cases suggests that the essential physics is already present for a single hole in an antiferromagnet, a limiting case that is reasonably well understood in terms of the 'string' picture. Following Ref. [4] we assume that 'charge spectra' in the low doping region are dominated by excitations of internal degrees of freedom of the spin bag like quasiparticles. More precisely, we assume that the quasiparticles in the low doping regime correspond to a hole oscillating rapidly (i.e. on an energy scale $\sim t$ ) within a region of enhanced spin disorder [8-10]. The entire quasiparticle (i.e. 'bare hole' plus 'defect region') 
then drifts slowly (i.e. on an energy scale $J$ ) and coherently through the system.

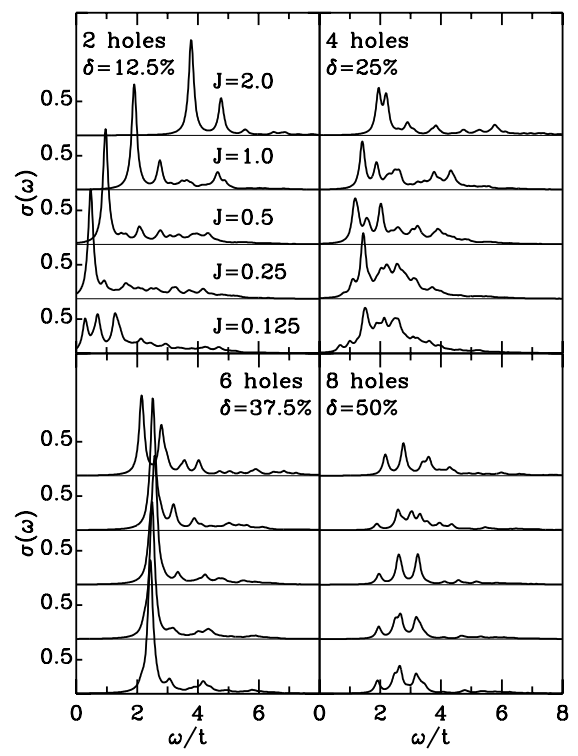

FIG. 3. Optical conductivity $\sigma_{x}(\omega)$ for $N=16$ and different hole numbers. Lorentzian broadening $0.1 t$.

A different way to express this picture would be 'incomplete spin charge separation', where the 'holon' (=bare hole) and the 'spinon' (=first spin defect created by the hopping hole) remain tied together firmly by a 'string', resulting in a bound state. As discussed in Ref. 任 both, the different energy scales of spin and charge correlation function and their different scaling behaviour with hole number $n_{h}$ can be understood immediately by assuming that the density operator couples only to the rapid incoherent motion, whereas the spin operator couples only to the slow coherent motion. Extending this line of thought we would expect that the current operator, which acts on charge degrees of freedom as well, should also couple to the rapid incoherent motion, i.e. excite 'internal degrees of freedom' of the spin bags. Due to the high symmetry for $\boldsymbol{q}=0$, however, there is predominantly one special type of state which is excited by the current operator: we may assume, that the drifting spin bag (or,phrased differently, the bound spinon-holon pair) to good approximation has maximum point group symmetry, i.e. it is an $s$-like state. The current operator for $\boldsymbol{q}=0$ is a vector, and hence must obey a 'dipole selection rule', in other words: it can couple only to ' $p$-like' excited states of the spin bag (or spinon-holon pair).

We make these considerations more quantitative, using the formalism of Ref. [9]. We begin with a hole created at some site $i$ in the Neel state. Acting with the hopping term, we obtain 'string states', where the hole is connected to its 'starting point' $i$ by a trace of misalligned spins [11. We denote a state with $\nu$ such defects as $|i, \nu, \mathcal{P}\rangle$ where $\mathcal{P}$ is shorthand for a set of numbers which parameterize the geometry of the hole path. The wave function for a hole which is trapped in the string potential around the site $i$ then reads

$$
\left|\Psi_{i, \lambda}\right\rangle=\sum_{\nu} \alpha_{\nu, \lambda} \sum_{\mathcal{P}} \phi_{\lambda}(\mathcal{P})|i, \nu, \mathcal{P}\rangle .
$$

Here, $\phi(\mathcal{P})$ denotes an extra phase factor, which determines the 'orbital symmetry' $\lambda$ of the self-trapped state. Let $\boldsymbol{e}$ denote a unit vector in the direction of the first step of the path $\mathcal{P}$. Then, $\phi_{\lambda}(\mathcal{P})=1$ will give a totally symmetric ('s-like') spin bag, $\phi_{\lambda}(\mathcal{P})=e_{x}$ will give a state with $p_{x}$-type symmetry etc. The coefficients $\alpha_{\nu, \lambda}$, which play the role of a 'radial wave function' associated with the orbital symmetry $\lambda$, are assumed to depend only on the length of the string, $\nu$. If we assume that the magnetic frustration in the system increases linearly with the string length $\nu$ 11], and introduce $\beta_{\mu, \lambda}=\alpha_{\mu, \lambda}(z-1)^{\mu / 2}$ (with $z$ the coordination number), the latter function can be determined from the (numerical) solution of the 'radial Schrödinger equation' 9]

$$
\begin{aligned}
-\frac{z}{z-1} \tilde{t} \beta_{1, \lambda}+2 J \beta_{0, \lambda} & =E_{\lambda} \beta_{0, \lambda} \\
-\tilde{t}\left(\beta_{\nu+1, \lambda}+\beta_{\nu-1, \lambda}\right)+J\left(\frac{5}{2}+\nu\right) \beta_{\nu, \lambda} & =E_{\lambda} \beta_{\nu, \lambda},
\end{aligned}
$$

where $\tilde{t}=\sqrt{z-1} t$. The normalization condition reads

$$
\beta_{0, \lambda}^{2}+\frac{z}{n_{\lambda}(z-1)} \sum_{\nu} \beta_{\nu, \lambda}^{2}=1,
$$

with $n_{s}=1, n_{p}=2$ and $\beta_{0, p}=0$. (and consequently the first equation (2) being omitted for $\lambda=p$ ). Next, a propagating spin bag with orbital symmetry $\lambda$ and momentum $\boldsymbol{k}_{0}$ would be described by

$$
\left|\Psi_{\lambda}\left(\boldsymbol{k}_{0}\right)\right\rangle=\sqrt{\frac{2}{N}} \sum_{j} e^{i \boldsymbol{k}_{0} \cdot \boldsymbol{R}_{j}}\left|\Psi_{j, \lambda}\right\rangle .
$$

When comparing with the numerical results we choose the 18-site cluster, where the single hole ground state momentum is $\boldsymbol{k}_{0}=(2 \pi / 3,0)$ (this avoids complications due to the spurious degeneracies in the $4 \times 4$ cluster). The little group of $\boldsymbol{k}_{0}$ comprises $E$ and the reflection by the $(1,0)$ direction, the ground state wave function being even. Choosing the current in $(1,0)$ direction the final states must be even as well and thus should correspond to a propagating $p_{x}$-like spin bag, whereas choosing the current in $(0,1)$ should couple to a propagating $p_{y}$-like spin bag. Assuming that the dispersion of the states (3) is solely due to the relaxation of the strings through the transverse part of the Heisenberg exchange and following Ref. [9] in the computation of the respective matrix elements, we obtain the dispersion of the $s$-like state as $E_{s}(\boldsymbol{k})=E_{s}+4 h_{s}\left(\left[\cos \left(k_{x}\right)+\cos \left(k_{y}\right)\right]^{2}-1\right)$, and that of a $p_{\alpha}$ state as $E_{p}(\boldsymbol{k})=E_{p}+2 h_{p} \cos \left(2 k_{\alpha}\right)$, with the 'spin-flip matrix element' $h_{\lambda}=\frac{J}{(z-1)^{n} \lambda} \sum_{\nu=0}^{\infty} \beta_{\nu, \lambda} \beta_{\nu+2, \lambda}$. The latter describes the truncation of the string by the 
Heisenberg exchange. Differences between those energies at $\boldsymbol{k}_{0}$ give the excitation energies in the optical conductivity. The matrix element of the current operator is $\left\langle\Psi_{s}\left(\boldsymbol{k}_{0}\right)\left|j_{\alpha}\right| \Psi_{p_{\alpha}}\left(\boldsymbol{k}_{0}\right)\right\rangle=-2 i t \sum_{\nu=0}^{\infty}\left(\alpha_{\nu, s} \alpha_{\nu+1, p}-\right.$ $\left.\alpha_{\nu, p} \alpha_{\nu+1, s}\right)$. Then, Figure 1 compares the current correlation function obtained by Lanczos diagonalization with the result of the string calculation for transitions to the lowest $p$-like state.

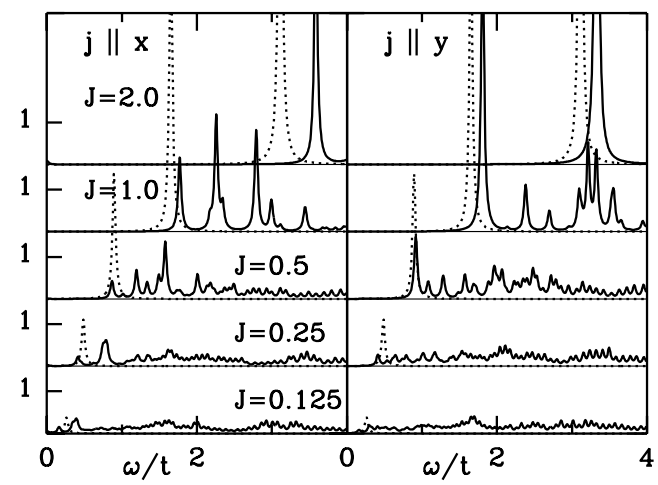

FIG. 4. Exact current correlation function for one hole in 18 sites (fulll line) compared to the result of the string calculation (dotted line). Lorentzian broadening $0.025 t$.

We note that the string calculation correctly reproduces the $J$-dependence of the offset. For larger $J$ and current perpendicular to the momentum, even the weight of the dominant peak is reproduced quite well. On the other hand, for smaller $J$ the 'peak' at the bottom of the Lanczos spectra seems to 'decay' into a multitude of small peaks, i.e. there is a strong damping. This is to be expected, because the $p$-like state first of all is high in energy (on the energy scale of spin fluctuations) and, having a node in the center, necessarily has a large spatial extent; most probably it is therefore very susceptible to scattering from spin excitations and/or finite size effects, so that the $p$-like 'state' rather has the character of a 'resonance'. While it seems that the string calculation reproduces the rapid decrease of spectral weight, any more quantitative comparison must fail due to this decay of the peak. Taking into account its highly oversimplified nature, the string calculation nevertheless predicts some trends reasonably well, and we believe that it actually is the correct explanation for the observed behaviour. It should be noted that a calculation for 'spinon'-'holon' pair, which is bound by a potential $\sim J$ times their distance would almost inevitably give an identical result. Finally, the apparent scaling of the dominant peak in the current-correlation function for $\boldsymbol{q}=(\pi / 3, \pi / 3)$ with $J$, which seems to occur for larger $J$, may be an indication that a propagating $p$-like spin bag represents an approximate eigenstate also for small finite momentua.

In summary, we have presented a systematical study of the optical conductivity in the $2 \mathrm{D} t-J$ model. We found that over the entire 'underdoped' range of hole concentrations, the optical spectrum is dominated by a single excitation at an energy of $\approx 1.7 \mathrm{~J}$. The appearance of the exchange constant $J$ as the energy scale of a 'charge spectrum' is not consistent with an interpretation in terms of spin charge separation, where the charge spectra would be described by the excitations of holons, whose energy scale is $t$; the latter would be neccessary to be consistent with the finite momentum current correlation and density correlation functions. On the other hand, an interpretation in terms of the 'string' picture, (or alternatively the assumption of tightly bound spinon-holon pair) where the dominant excitation in $\sigma(\omega)$ is interpreted as transition to the lowest excited state of the 'spin bag' with $p$-type symmetry, readily provides a semiquantitative explanation for the numerical data. The absence of an appreciable finite frequency component in $1 D$ then fits nicely with this picture: in $1 D$ spinon and holon truly unbind, so that excited 'bound states' which could serve as final states in $\sigma(\omega)$ do not exist.

We note that 'incomplete spin-charge separation', i.e. the formation of tightly bound spinon-holon pairs, in reality would imply absence of spin charge separation. When probed with wave lengths larger than its spatial extent, or very low energies, the spinon-holon pair should behave just like a spin-1/2 Fermion, so that the 'effective' low energy theory would be a Fermi liquid of quasiparticles corresponding to the doped holes. This scenario is indeed suggested by diagonalization [12,13, and other [14,15 studies. The nature of the 'transition' at higher doping levels, and the resulting ground state remains to be clarified.

Financial support of R. E. by the European Community and of P. W. by KBN is most gratefully acknowledged.

[1] J. Uchida et al. Phys. Rev. B 43, 7942 (1991).

[2] I. Sega and P. Prelovshek, Phys. Rev. B 42, 892 (1990).

[3] A. Moreo and E. Dagotto Phys. Rev. B 42, 4786 (1990).

[4] R. Eder et al., Phys. Rev. Lett. 74, 5124 (1995).

[5] E. Dagotto at al., Phys. Rev. B 45, 10741 (1992).

[6] B. Batlogg et al., Physica C 235-240, 130 (1994).

[7] R. Eder and Y. Ohta, Phys. Rev. B 51, 11683 (1994);

[8] J. R. Schrieffer et al., Phys. Rev. Lett. 60, 944 (1988).

[9] R. Eder and K. W. Becker, Phys. Rev. B 44, 6982 (1991).

[10] J. Gan and P. Hedegard, Phys. Rev. B 53, 911 (1996).

[11] L. N. Bulaevskii et al., Sov. Phys. JETP 27836 (1968).

[12] R. Eder, Y. Ohta, and T. Shimozato, Phys. Rev. B 50, 3350 (1994); R. Eder and Y. Ohta, Phys. Rev. B 51, 6041 (1994).

[13] J. Jaklic and P. Prelovshek, cond-mat/9603081.

[14] X. G. Wen and P. A. Lee, Phys. Rev. Lett. 76, 503 (1996).

[15] E. Dagotto et al., Phys. Rev. Lett. 73, 728 (1994). 